
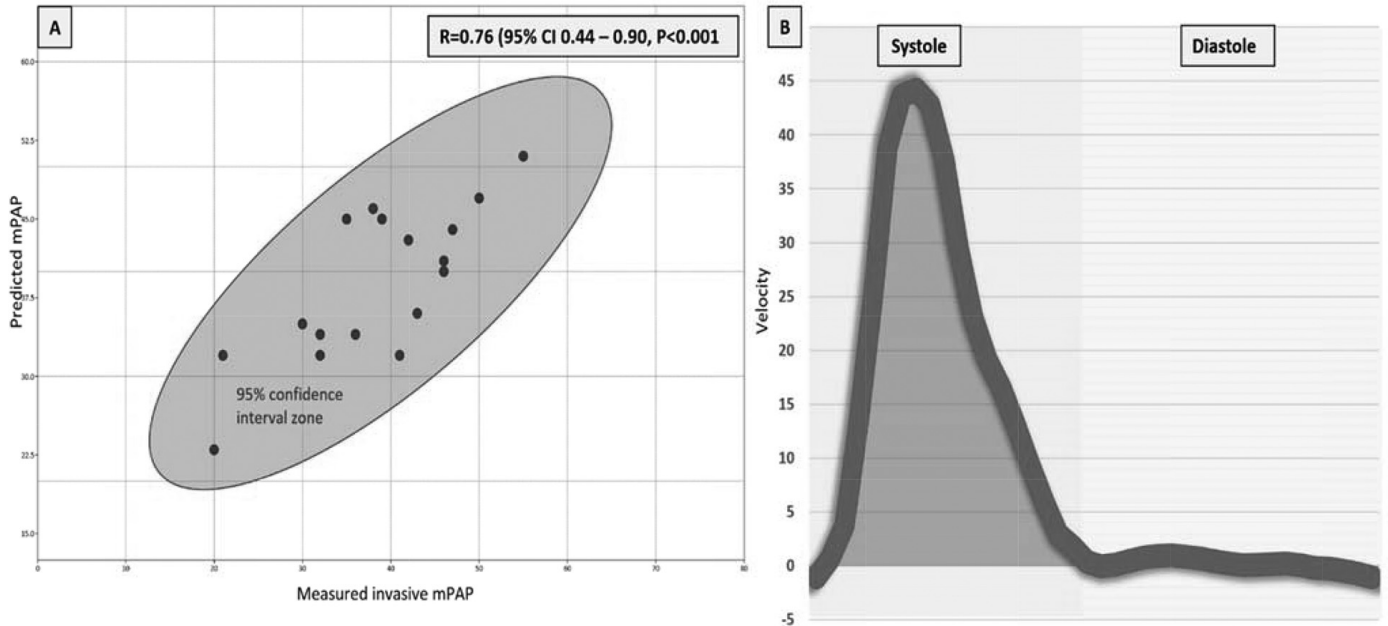

Abstract 26 Figure 1 Panel A: scatter plot of measured and predicted mean pulmonary artery pressure. Panel B: Main pulmonary artery (MPA) time-resolved, flow curves (the light blue area was used to compute mean pan-systolic area)

\begin{tabular}{llllll}
$\begin{array}{l}\text { Abstract 26 Table } 1 \\
\text { the study }\end{array}$ & \multicolumn{5}{l}{ Summary of CMR parameters evaluated in } \\
\hline \multicolumn{5}{l}{ Male (n=8) } & \multicolumn{4}{l}{ Female (n=10) } \\
\hline & Mean & SD & Mean & SD & P-value \\
\hline Age (Years) & 71.0 & 6.8 & 66.0 & 7.8 & 0.16 \\
Invasive mPAP (mmHg) & 36.4 & 9.5 & 41.9 & 8.9 & 0.23 \\
RA Area (cm ${ }^{2}$ ) & 23.7 & 6.1 & 28.1 & 11.8 & 0.31 \\
RVEDV (ml) & 165.3 & 51.1 & 202.3 & 72.6 & 0.22 \\
RVESV (ml) & 114.8 & 49.1 & 138.0 & 58.7 & 0.37 \\
RVSV (ml) & 50.7 & 25.5 & 64.4 & 22.7 & 0.25 \\
RVEF (\%) & 32.5 & 15.7 & 33.2 & 8.9 & 0.91 \\
PA SV (ml) & 50.0 & 24.7 & 53.6 & 16.8 & 0.72 \\
PA Mean Systolic & 15.0 & 6.9 & 14.3 & 3.4 & 0.79 \\
Velocity (cm/s) & & & & & \\
PA WSR & 39.5 & 22.1 & 36.6 & 10.2 & 0.74 \\
PA WSS & 138.1 & 77.3 & 127.9 & 35.2 & 0.73 \\
Predicted mPAP CMR & 37.5 & 7.4 & 40.5 & 6.8 & 0.40 \\
MODEL (mmHg) & & & & & \\
\hline
\end{tabular}

acquisition. The velocity encoded images were analysed for the following: mean pulmonary artery pan-systolic velocity (PASV), MPA stroke volume, MPA wall shear stress (WSS) and wall shear rate (WSR). The 4-chamber cine was used to measure end-diastolic right atrial area. Right ventricular volumes were analysed using standard methods. Stepwise multiple regression model of significantly associated parameters $(\mathrm{p}<0.05)$ was developed.

Results Mean age of the 18 patients was $68.78 \pm 7.46$ years (44\% males). The following CMR metrics demonstrated significant association to the measured mPAP: RA area $(r=0.65$ $\mathrm{p}=0.03)$; MPA mean pan-systolic velocity $(\mathrm{r}=-0.57 \mathrm{p}=0.01)$; RVEDV ( $\mathrm{r}=0.52 \mathrm{p}=0.03)$; RVEF $(\mathrm{r}=-0.40 \mathrm{p}=0.10)$; RVESV $(\mathrm{r}=0.58 \mathrm{p}=0.01)$. In stepwise multiple regression, only two parameters demonstrated independent association to mPAP RA Area and MPA mean pan-systolic velocity. The predicted mPAP demonstrated good correlation to the measured mPAP $(\mathrm{R}=0.76, \mathrm{p}<0.001)$.

Conclusion(s) Mean pulmonary artery systolic velocity and right atrial area are independently associated with mPAP.
Our novel CMR prediction model for mPAP, comprising of these two metrics, demonstrates high association to the measured mPAP by invasive haemodynamic study.

\section{MIXED VENOUS OXYGEN LEVELS IN PULMONARY HYPERTENSION IS ASSOCIATED WITH RIGHT HEART MULTI-PARAMETRIC ASSESSMENT}

Natasha Barker, Benjamin Fidock, Nithin Balasubramanian, Alistair W Macdonald, David Capener, Christopher S Johns, Kavitasagary Karunasaagarar, Graham Fent, Abdallah Al-Mohammad, Alexander Rothman, David G Kiely, James M Wild, Andrew Swift, Pankaj Garg. The University of Sheffield, Sheffield University teach hospitals NHS foundation trust

\subsection{6/heartjnl-2019-BSCMR.27}

Background Pulmonary hypertension (PH) is debilitating disease characterized by a progressive increase in pulmonary arterial pressure (PAP) that leads to right ventricular (RV) failure and death. Mixed venous oxygen saturation (SVO2) represents the oxygen saturation of blood returning to the lungs before reaching the alveolo-capillary units. SVO2 is strongly associated with clinical outcomes in $\mathrm{PH}$. The relationship of noninvasive CMR metrics to this prognostically relevant parameter in patients with $\mathrm{PH}$ are unknown.

Purpose This study sought to develop an early understanding of which CMR volumetric and flow parameters are most associated with SVO2.

Methods Eighteen $(n=18)$ patients were prospectively recruited at a large tertiary $\mathrm{PH}$ unit. The $\mathrm{SVO} 2$ was measured during right heart catheterisation. All patients had CMR on the 1.5 $\mathrm{T}$ scanner (HDx scanner, GE Healthcare, Waukesha, Wisconsin, USA), using an 8-channel cardiac coil. Subjects were scanned in the supine position with electrocardiogram (ECG) gating. CMR protocol included long and short axis cines and through-plane pulmonary artery phase contrast acquisition. The velocity encoded images were analysed for the following: mean pulmonary artery (MPA) systolic velocity, MPA stroke volume, MPA wall shear stress (WSS) and wall shear rate (WSR). The 4 chamber cine was used to measure end-diastolic right atrial (RA) area. RV volumes were analysed using standard methods. Stepwise multiple regression model of significantly associated parameters $(\mathrm{p}<0.05)$ was developed. 


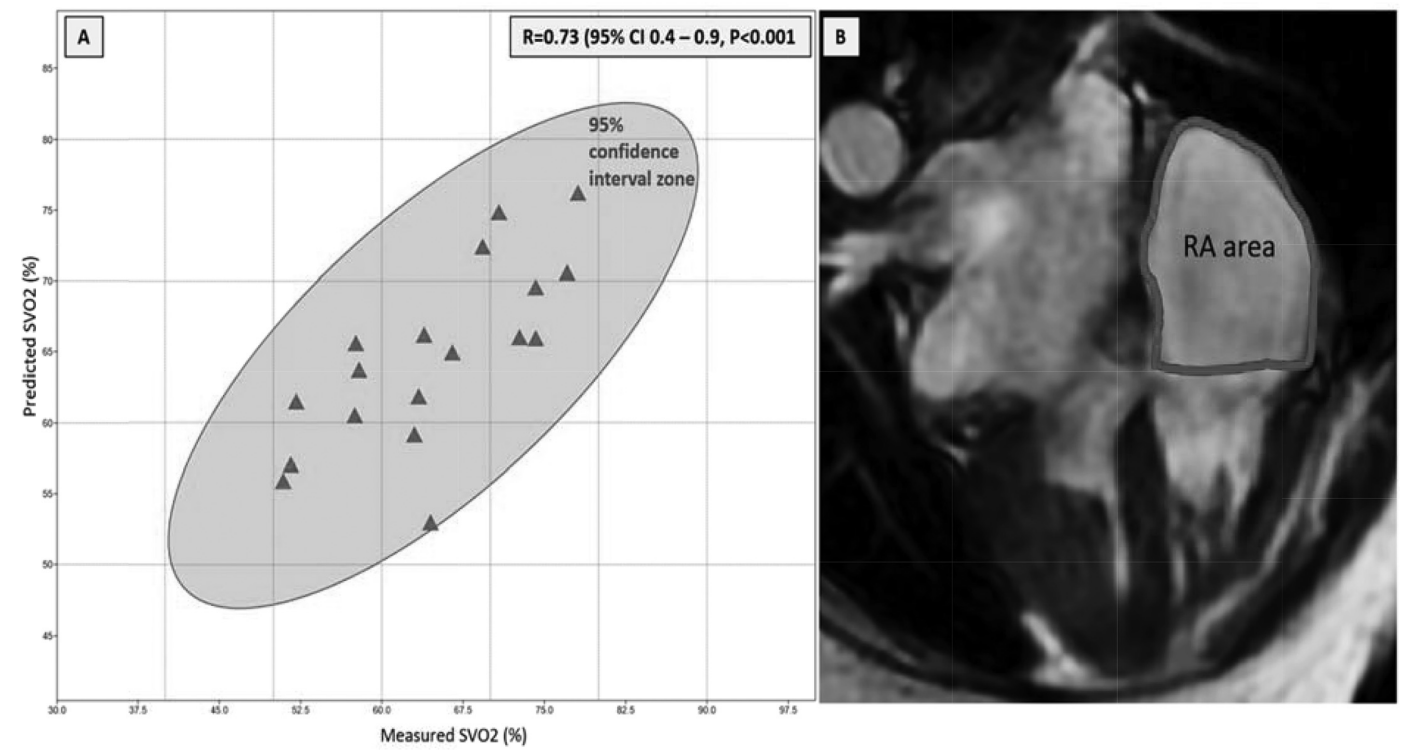

Abstract 27 Figure 1 Panel A: scatter plot of measured and predicted SV02. Panel B: Right atrial size was computed in 4-chamber view at end-systole (RV), just before the opening of the tricuspid valve

\begin{tabular}{lllll}
$\begin{array}{l}\text { Abstract 27 Table } 1 \\
\text { the study }\end{array}$ & Summary of CMR parameters evaluated in \\
\hline CMR parameters & Mean & SD & $\begin{array}{l}\text { Pearson } \\
\text { Correlation } \\
\text { (P-value) }\end{array}$ & $\begin{array}{l}\text { Regression } \\
\text { model }^{*}\end{array}$ \\
& & & $-0.57(0.01)$ & F-ratio=8.56 \\
\hline RA Area* (cm2) & 25.64 & 9.05 & $0.5(0.03)$ & p $<0.01$ \\
PA SV* (mls) & 51.58 & 21.05 & $0.42(0.08)$ & \\
LVEDV (mls) & 119 & 39.62 & $0.22(0.38)$ & \\
LVESV (mls) & 54.92 & 16.57 & $0.42(0.08)$ & \\
LVSV (mls) & 63.39 & 29.97 & $0.27(0.3)$ & \\
LV mass (grams) & 82.61 & 22.03 & $0.26(0.29)$ & \\
LVEF (\%) & 53.02 & 10.34 & $-0.12(0.63)$ & \\
RVEDV (mls) & 181.72 & 62.52 & $-0.30(0.22)$ & \\
RVESV (mls) & 125.08 & 53.26 & $-0.35(0.16)$ & \\
RVSV (mls) & 56.78 & 24.59 & 0.39 & \\
RVEF (\%) & 32.81 & 12.79 & $0.46(0.05)$ & \\
\hline
\end{tabular}

Results The left ventricular and RV volumetric functional parameters demonstrated no association to SVO2 $(\mathrm{p}>0.05)$ in any of the participants. However, a negative association was shown between RA area and SVO2 $(\mathrm{R}=-0.57, \mathrm{p}=0.01)$. The only other parameter which correlated with SVO2 was MPA stroke volume $(\mathrm{R}=0.5, \mathrm{p}=0.03)$. In stepwise multiple regression, both parameters demonstrated independent association to SVO2. The predictive values generated by a combined model demonstrated high correlation to measured SVO2 $(\mathrm{R}=0.73$, $\mathrm{p}<0.001)$.

Conclusion(s) RA area and MPA stroke volume are independently associated with SVO2. A novel CMR prediction model comprised of these two metrics, demonstrates high association to the measured SVO2 by invasive haemodynamic study. Further work to determine reproducibility and reliability is needed before this method becomes an important prognostic tool for $\mathrm{PH}$ patients. 